\title{
An Application of the Acoustic Similarity Law to the Numerical Analysis of Centrifugal Fan Noise*
}

\author{
Wan-Ho JEON ${ }^{* *}$, Duck-Joo LEE ${ }^{* * *}$ and Huinam RHEE ${ }^{* * * *}$
}

\begin{abstract}
Centrifugal fans, which are frequently used in our daily lives and various industries, usually make severe noise problems. Generally, the centrifugal fan noise consists of tones at the blade passing frequency and its higher harmonics. These tonal sounds come from the interaction between the flow discharged from the impeller and the cutoff in the casing. Prediction of the noise from a centrifugal fan becomes more necessary to optimize the design to meet both the performance and noise criteria. However, only some limited studies on noise prediction method exist because there are difficulties in obtaining detailed information about the flow field and casing effect on noise radiation. This paper aims to investigate the noise generation mechanism of a centrifugal fan and to develop a prediction method for the unsteady flow and acoustic pressure fields. In order to do this, a numerical analysis method using acoustic similarity law is proposed, and it is verified that the method can predict the noise generation mechanism very well by comparing the predicted results with available experimental results.
\end{abstract}

Key Words: Centrifugal Fan Noise, Acoustic Similarity Law

\section{Introduction}

Noise generated from centrifugal fans, which are widely used in fluid machines, is becoming more serious problems as the required power and the rotating speed of impeller increase. Although some limited researches on the noise source identification and analyses have been performed since early 1960's, they are still not enough to establish a systematic methodology that can be actually implemented for the design of centrifugal fans. On the contrary, the axial fans, which are used in aircraft engines, therefore, noise generated from them is concerned very much, have been studied very much. One of the reasons that the centrifugal fans have not been studied enough is that the analysis method of the flow field was not established well and the complicated scattering effect of casing should be considered in the calculation.

In this paper, we develop a noise analysis method for

* Received 20th February, 2003 (No. 03-5017)

** Head of Flow Noise Research Lab., ECM Co., Ltd., 1009 Byuksan Digital Valley II, Kasan-dong, Kumchongu, Seoul, Korea

*** Department of Mechanical Engineering, KAIST, 373-1 Kusung-dong, Yusung-ku, Taejon, Korea

**** School of Mechanical and Automotive Engineering, Sunchon National University, 315 Maegok-Dong, Sunchon, Junnam 540-742, Korea. E-mail: hnrhee@sunchon.ac.kr centrifugal fans and apply it to an example case, for which experimental results are available. For this purpose, an acoustic prediction method is developed to analyze the centrifugal fan noise. The predicted results are then converted appropriately by using the acoustic similarity law so that they can be compared with the experimental data.

Previous studies on the centrifugal fans noise were mainly about the noise reduction method and the development of experimental methodology ${ }^{(1)-(6)}$. Some examples of the noise reduction method are as follow ; increasing cutoff distance ${ }^{(1)-(3)}$, tilting impeller blade and cutoff $^{(1)-(4)}$, placing meshes in the front and back of blades $^{(1),(2)}$, arranging blades asymmetrically ${ }^{(1),(2)}$, and using active noise control method ${ }^{(1),(2),(5),(6)}$, etc. However, these methods do not provide information on noise source and noise generation mechanism, but only suggest noise reduction techniques.

Madison performed a study on the experimental method of centrifugal fan noise in $1949^{(7)}$. In 1963 , Maling proposed a simple equation by the dimensional analysis using Buckingham PI theorem for broadband noise of a centrifugal blower with forward curved blades $^{(8)}$. Chanaud claimed that the centrifugal fan noise has dipole characteristics by conducting experiments for a fan having forward curved blades ${ }^{(9)}$. Weidemann initiated researches on acoustic similarity law extensively ${ }^{(10)}$. 
Weidemann expressed the noise as a product of several nondimensional parameters and functions of them, therefore, divided the noise into sound generation and radiation components. In 1975, Neise applied the similarity law using Weidemann's result to the analysis of noise at BPF (blade passing frequency) and its higher harmonics $^{(7)}$. Neise also applied it to the broadband noise in $1982^{(11)}$. Since then, researches on rotating stall and broadband noise have been performed by using the similarity law ${ }^{(12)-(14)}$.

In this study we analyze flow field to obtain the force variations, and then calculate the acoustic pressure using the one of the acoustic analogy. We use the discrete vortex method to calculate the flow field, and then predict the noise caused by the unsteady pressure fluctuations at the impeller blades using the force (or pressure) information calculated from the flow field. In order to do this, forces acting on elements of the impeller blades are calculated, and then the acoustic field information at far-field caused by moving point forces is predicted by using Lowson's method. Lowson's method can be used to predict acoustic field when a dipole moves at an infinite boundary, therefore, cannot consider scattering and diffraction. However, Lowson's method can be easily used for the noise source identification and for the prediction of acoustic pressures $^{(15),(16)}$. In this paper, for the Weidemann's centrifugal fan, the flow field analysis and acoustic pressure field prediction are performed. These numerical results are then compared with the experimental results, and also the acoustic similarity law is applied to tonal noise.

\section{Numerical Method}

\subsection{Non-dimensional parameters}

Strauhal number $(\mathrm{St})$ is the most important parameter related to noise generation, and defined generally as follows :

$$
S t=\frac{f D}{U},
$$

where $D$ is the characteristic length of the object and $U$ is the flow velocity. $f$ is the frequency of the sound generated from cylinders subjected to a flow. St is a nondimensionalized form of the frequency, $f$. However, for a centrifugal fan in this study, the definition of $S t$ is modified as below;

$$
S t=\frac{\pi}{Z} \cdot \frac{f D}{U}=\frac{f \hat{D}}{U},
$$

where $Z$ is the number of blades. Equation (2) means that the characteristic length is $\hat{D}(=\pi D / Z)$, and that $S t=1$ for the BPF and $S t=2$ for the first harmonic, etc.

Another important non-dimensional variable used in this study is the Helmholtz number $(\mathrm{He})$ which is defined as follow:

$$
H e=D / \lambda=f D / a_{0}=\frac{Z}{\pi} M a S t,
$$

where $\lambda, a_{0}$ and $M a$ are the wave length, speed of sound and Mach number, respectively. $\mathrm{He}$ is not related to the sound generation mechanism, but is related to the directivity of sound and the resonance of the object. He is derived from the non-dimensionalization of the resonance frequency of Helmholtz resonator.

\subsection{Similarity law of the centrifugal fan noise}

In fact past, without information on the flow field, the noise generated by a centrifugal fan could be identified only by measurements using prototypes. However, similarity law makes predictions of noise possible by using database of noise measured from centrifugal fans of similar design under similar operating conditions. The similarity law has had a much contribution to the identification and understanding of noise sources by centrifugal fans, and is still being studied in various application areas.

In 1970, Weidemann performed a study on the similarity law based on measurement data from impellers of various diameters $^{(10)}$. Weidemann experimented impellers with backward curved blades and without casing in a free field. A rectangular type wedge was used to generate tonal sounds. Weidemann obtained the following relationship for the acoustic pressure level $K$ :

$$
K=F\left(M a, R e, S t, x_{i} / D, \phi\right),
$$

where $R e$ is Reynolds number and $\phi$ is the flow coefficient. $K=K_{d}=\Delta \tilde{P} / P_{0}$ for the discrete frequency sound. $\Delta \tilde{P}$ is the root mean square value of measured acoustic pressure, and $P_{0}$ is the reference pressure. $x_{i} / D$, which is the measurement position, and flow coefficient, $\phi$, are constant. Equation (4) only contains terms related to the sound generation, therefore, another term in $\mathrm{He}$ needs to be included as a form of system frequency response function. This function is represented as $\mathrm{G}(\mathrm{He})$, and has physical meanings of the resonance effect on the acoustic pressure level and of the radiation direction at a certain frequency. Resultantly, the final form for the acoustic pressure level is obtained as below ${ }^{(10)}$ :

$$
K=M a^{\alpha} \operatorname{Re}^{\beta} F(\mathrm{St}) G(\mathrm{He})
$$

In Eq. (5), first two non-dimensional parameters are related to the impeller tip speed, viscous effect and effect of impeller diameter. $F(S t)$ represents the spectral distribution of the generated sound. $\mathrm{G}(\mathrm{He})$ is related to the geometrical shape. In other words, $M a^{\alpha}, R e^{\beta}$ and $F(S t)$ are related to the sound generation while $G(\mathrm{He})$ is related to the sound radiation characteristics and resonance.

Based on the experimental results by Weidemann, $\alpha_{d}=2.6$ and $\beta_{d}=0.2$ for tonal sounds. For tonal sounds, the radiated acoustic power $W$ is as follows:

$$
W \sim U^{5.6} D^{2.4}
$$

The advantage of Weidemann's equation is that the fan noise is divided into sound generation and radiation parts. Some studies followed Weidemann's works to find 
$F(S t)$ and $G(H e)$. In 1975, Neise studied the similarity law for the noise at BPF and its higher harmonics using Weidemann's result by experimental works ${ }^{(7)}$. Neise confirmed the characteristics of $F$ and $G$, and also the fact that $W$ is proportional to $U^{5.6}$. Neise also performed a research on the broadband noise of centrifugal fans by using the similarity law in $1982^{(11)}$. We will use the same form as Eq. (5) for the similarity law in this paper.

As a summary, similarity law is used to predict noises from centrifugal fans of different design under different operating conditions by applying Eq. (5). The purpose of this paper is to obtain $F$ numerically, and then compare the predicted results with the experimental data in order to verify the similarity law. The physical system that this paper deals with is shown in Fig. 1. Tonal noise is mainly concerned in this paper because they are governing in this type of configuration rather than broadband noise.

\subsection{Flow field analysis of a centrifugal fan}

Discrete Vortex Method (DVM) is used to analyze the flow. DVM is generally used for the unsteady flow analysis. It has been applied to the flow field analysis of centrifugal fans ${ }^{(15)-(17)}$. The centrifugal fan studied in this paper (Fig. 1) is identical with the Weidemann's fan, which has an impeller and a rectangular wedge. The inner and outer diameters are $0.122 \mathrm{~m}$ and $0.28 \mathrm{~m}$, respectively. The inlet and outlet angles are $23.4^{\circ}$ and $33.5^{\circ}$, respectively. The gap between the impeller and wedge is $0.0028 \mathrm{~m}$. The impeller operates at a constant flow coefficient, which is as follow:

$$
\phi=\frac{Q}{\pi D b V_{\text {tip }}} \approx 0.14,
$$

where $b$ and $D$ are the diameter and width of the impeller.

It is assumed that the flow is two dimensional, unsteady and invicid. The impeller blades are modeled as discrete vortices. The impeller rotates at a constant rate. Wake vortices are shed at the trailing edge of blades at every time step, and then convected. Wedge is modeled as a source panel. Based on these assumptions and models, the induced velocity at a control point $\left(\vec{x}_{c j}\right)$ is as follow:

$$
\vec{U}\left(\vec{x}_{c} ; t\right)_{j}=\vec{U}_{Q}\left(\vec{x}_{c} ; t\right)_{j}+\vec{U}_{b v}\left(\vec{x}_{c} ; t\right)_{j}
$$

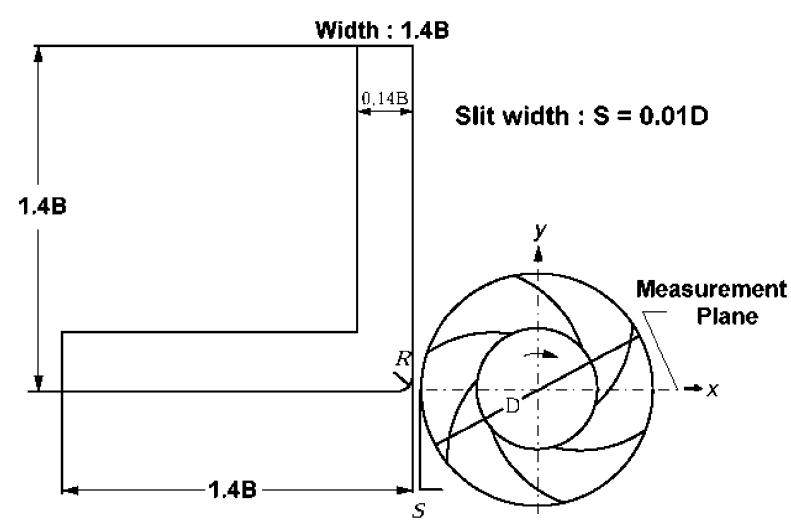

Fig. 1 Dimensions of the impeller and wedge

$$
+\vec{U}_{w v}\left(\vec{x}_{c} ; t\right)_{j}+\vec{U}_{s p}\left(\vec{x}_{c} ; t\right)_{j}
$$

The four right-hand side terms represent velocities induced by point source, bound vortices, wake vortices, and source panel, respectively ${ }^{(15)}$. The strengths of the bound and wake vortices, and the source panel can be calculated with the boundary condition that the normal component of the velocity at $\vec{x}_{c j}(j=1,2, \cdots, n c)$, where $n c$ is the number of elements for each blade, is zero and with Kelvin's condition, which means the conservation of total circulation $^{(15),(16)}$. The convection of the vortices shed at the trailing edge is calculated by 2nd order Runge-Kutta method. The forces on the blades are calculated by the unsteady Bernoulli's equation $^{(15)}$.

\subsection{Calculation of acoustic field of a centrifugal fan}

Strong tonal noises are generated in the system in Fig. 1 by the interaction between the rotating impeller and the wedge, which locate closely each other. Therefore, the noise can be predicted with the information on the unsteady force fluctuations. With the assumption that the dipole noise is governing, the following Lowson's equation, which predicts acoustic field generated by a moving point force, is used ${ }^{(17)}$ :

$$
\rho-\rho_{0}=\left[\frac{x_{i}-y_{i}}{4 \pi a_{0}^{3} r^{2}}\left\{\frac{\partial F_{i}}{\partial t}+\frac{F_{i}}{1-M_{r}} \frac{\partial M_{r}}{\partial t}\right\}\right],
$$

where $F_{i}$ is the force and $r$ is the distance between the observer and noise source. $\rho$ is the fluid density. $x$ and $y$ are the positions of observer and noise source, respectively. $M_{r}$ is defined as below ${ }^{(17)}$ :

$$
M_{r}=\frac{\left(x_{i}-y_{i}\right)}{r} M_{i}
$$

The relationship between the density and pressure is as below:

$$
P=a_{0}^{2} \rho
$$

The first and second terms in the right hand side of Eq. (9) are the acoustic pressures by unsteady force fluctuations and accelerations (rotations), respectively. Since Eq. (9) calculates the acoustic pressure field by a moving point force on an element of a blade, the total acoustic pressure field can be calculated by the superposition of effects by all elements.

\section{Numerical Results}

\subsection{Flow field of a centrifugal fan}

Numerical analysis is performed for various rotating speed from $1200 \mathrm{rpm}$ to $4100 \mathrm{rpm}$. The calculations are made up to 20 non-dimensional time. One nondimensional time represents one revolution of the impeller. The head indicating the performance of the fan are also calculated as shown in Fig. 2. The heads show severely unsteady states. As the rotating speed increases, the mean head increases and the fluctuations also increase. 
Since the noise is caused not by the mean head, but by the fluctuations, therefore, we can easily anticipate that the noise will increase as the rotating speed of the impeller increases. In Fig. 2 we can see that the transient states continue up to approximately 3 non-dimensional time, therefore, we should use the flow field data for after 3 nondimensional time. Figure 3 shows the distribution of shed vortices at different times for $3000 \mathrm{rpm}$ case. In Fig. 3, circles and triangles represent vortices rotating at clockwise (cw) and counter-clockwise (ccw) directions, respectively. The size of symbols means the strengths of the vortices. The impeller rotates at ccw direction. It is noted that when the impeller blade passes wedge the direction of vortices changes from $\mathrm{cw}$ to $\mathrm{ccw}$, and the strength increases. These phenomena are periodic, that is, 6 times per a revolution. The unsteady pressure head fluctuations are caused by this interaction. In Fig. 3, it can be seen that the distribution of vortices is stabilized except near wedge at 12 non-dimensional time.

\subsection{Noise analysis of a centrifugal fan}

Weidemann measured noise data at $3000 \mathrm{rpm}$ as shown in Fig. 4. Figure 4 presents strong peaks at $300 \mathrm{~Hz}$ and its harmonic frequencies, therefore, tells that tonal noise is governing. With the flow field data for from 4 to

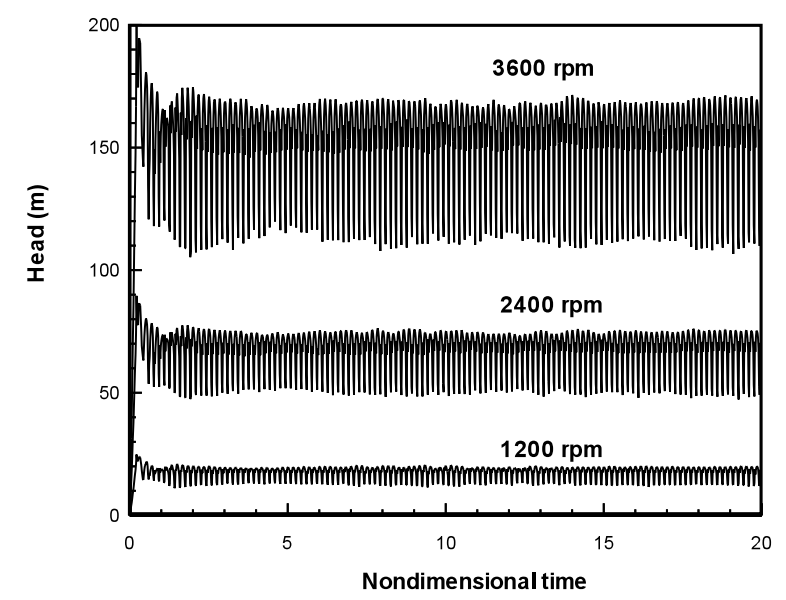

Fig. 2 Variations of head with time at different rpm

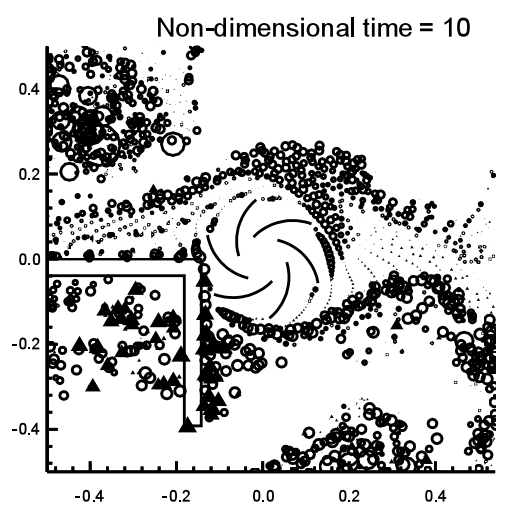

20 non-dimensional time, the acoustic pressure at the observer point is predicted by Lowson's equation. Figure 5 shows the calculated acoustic pressures at $3000 \mathrm{rpm}$ both in time and frequency domains. The locations and magnitudes of the peaks in Figs. 4 and 5 (b) are consistent very well. However, from the 6th peak, the calculated magnitude in Fig. 5 (b) is smaller than the experimental value in Fig. 4. Therefore, the similarity law is constructed by considering up to only 5 th peak in his paper. In order to determine the exponent of Ma on Eq. (5), the overall sound pressure level (SPL) is calculated for various rotating speeds in Fig. 6. Here, the components below $120 \mathrm{~Hz}$ are not considered to compare with the experimental results. Figure 6 shows that the overall SPL is proportional to $U^{2.8}$ by both calculation and experiment. This consistency verifies that the numerical calculation can predict acoustic pressure for varying rpm case as well as a constant rpm case. We use the experimental value 0.2 as the exponent of $R e$. Therefore, the non-dimensionalized tonal acoustic pressure $K_{d}$ is expressed as:

$$
K_{d} \sim M a^{2.6} R e^{0.2}
$$

\subsection{Application of the similarity law to the cen- trifugal fan noise}

This paper deals with tonal noises related to BPF

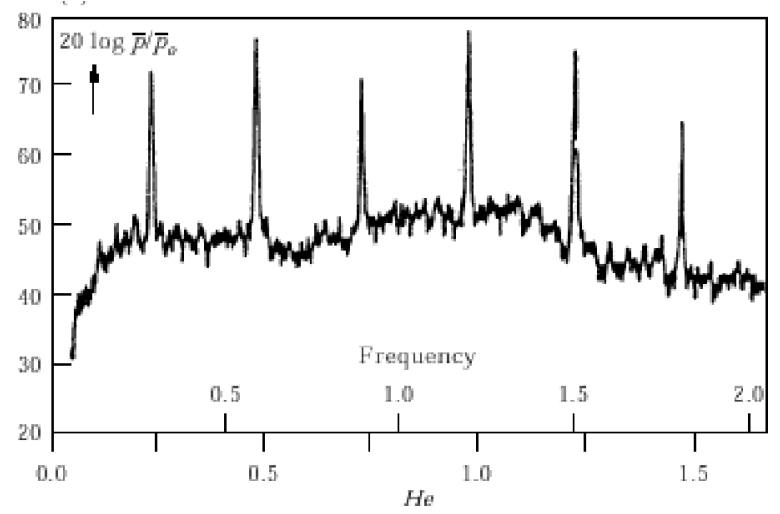

Fig. 4 Measured noise spectrum by Weidemann ${ }^{(10)}$. Rotating velocity is $3000 \mathrm{rpm}(\mathrm{BPF}: 300 \mathrm{~Hz})$.

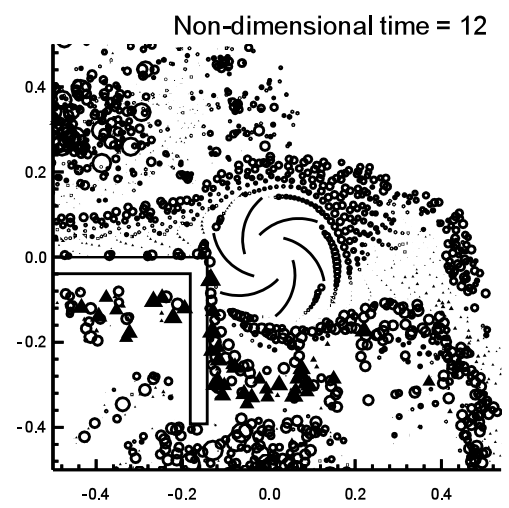

Fig. 3 Distribution of shed vortices with time (3000 rpm) 


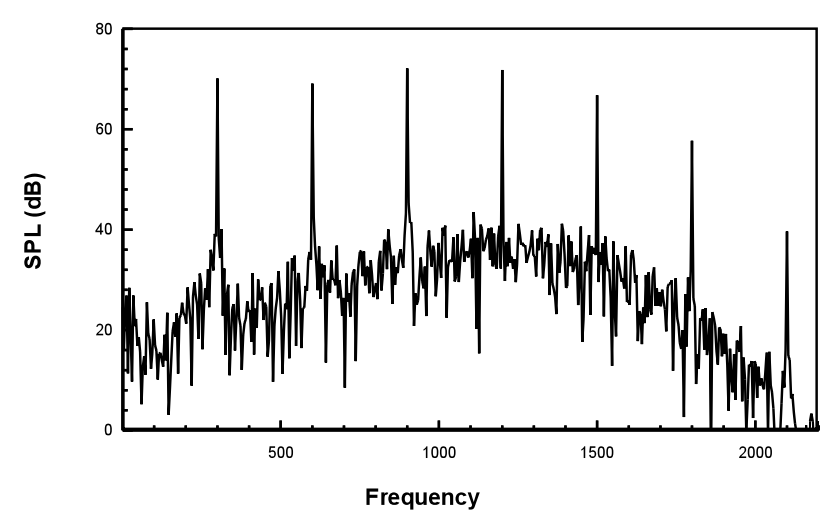

(a) Time signal of the calculated sound pressure

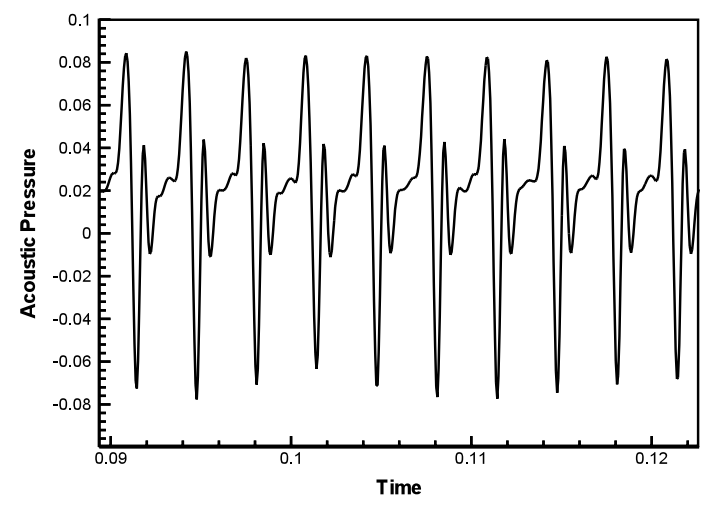

(b) FFT of the calculated sound pressure

Fig. 5 Predicted sound pressure at $3000 \mathrm{rpm}$

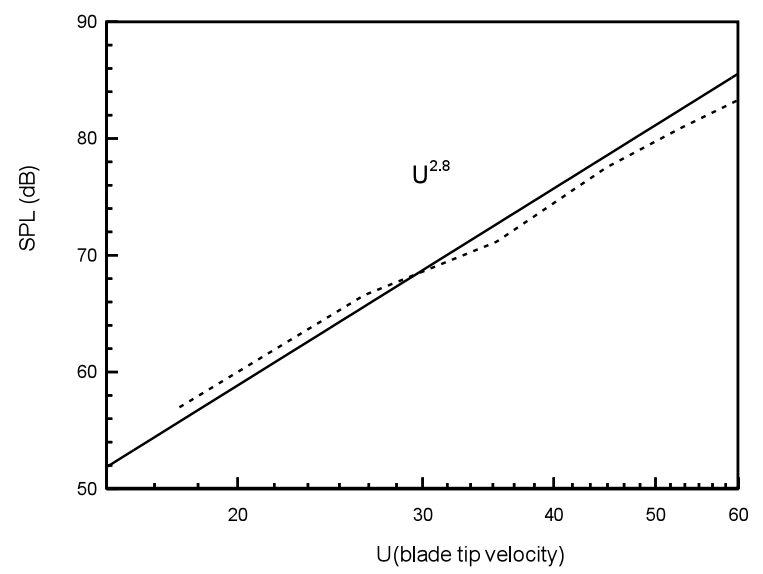

Fig. 6 Determination of the exponent of Mach number (Dashed: Calculated)

and its harmonics, therefore, we construct Fig. 7 which shows the trajectories of tonal peaks with varying rpms from 1200 to 4 100. In Fig. 7 the frequency contents for $3000 \mathrm{rpm}$ case are also shown for an explanation. We can see that the trends of five curves for different $S t$ numbers are consistent. The equal-St curves can be transformed to similar-level curves by subtracting the level differences of the curves. This results in the curves shown in Fig. 8 (a). In Fig. 8 (a), all curves are almost overlapped within $\pm 3 \mathrm{~dB}$ error range, and hereafter the representative curve is called

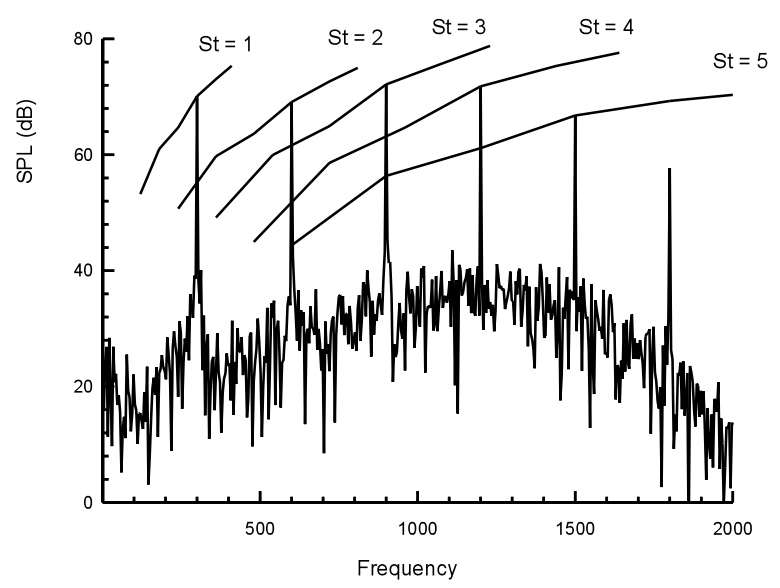

Fig. 7 Sound pressure levels at peak frequencies by changing rotating rpm

$F_{2}(H e)$ function. This curve is almost same as the $H e^{2.6}$ curve as shown in Fig. 8 (a). $F_{2}(H e)$ can be approximately expressed as $\mathrm{He}^{2.6}$.

$$
H e^{2.6} \sim M a^{2.6} S t^{2.6}
$$

Now, the system frequency characteristics, $G$ function is defined as follow:

$$
G_{d}(H e)=\frac{F_{2}(H e)}{H e^{2.6}}
$$

The $G$ function, shown in Fig. 8 (b), is also called the acoustic frequency response function, and has a meaning of resonance that can be occurred in a laboratory room during the experiment, and directivities. This paper concerns sound radiation in a free field, therefore, scattering, diffraction and resonance effects can not be considered. So the $G$ function looks almost flat in Fig. 8(b). Figure 9 shows the $F_{1}(S t)$ function, which is the level difference of average values of $S t=$ const. curves. In Fig. 9, numerical analysis and experiments show consistent behavior except that the calculated one is a little higher than the experimental result for $S t=2$ and 3. This deviation is more noticeable in $F(S t)$, shown in Fig. 10, calculated as below:

$$
F_{d}(S t)=\frac{F_{1}(S t)}{S t^{-2.6}}
$$

$F$ function is called spectral distribution function and means sound generation. The reason the calculated value is higher for $S t=2$ and 3 is that the flow field analysis methodology used in this study over predicts the unsteady condition and that the three dimensional geometry is assumed as two dimensional. However, the amount of over prediction is actually small, i.e., only $1 \mathrm{~dB}$.

The acoustic pressure is proportional to the product of the two functions, i.e.,

$$
K_{d} \sim F_{1}(S t) F_{2}(H e)
$$

By substituting Eqs. (14) and (15) into Eq. (16) the following equation is obtained:

$$
K_{d} \sim S t^{-2.6} F_{d}(S t) M a^{2.6} S t^{2.6} G_{d}(H e)
$$




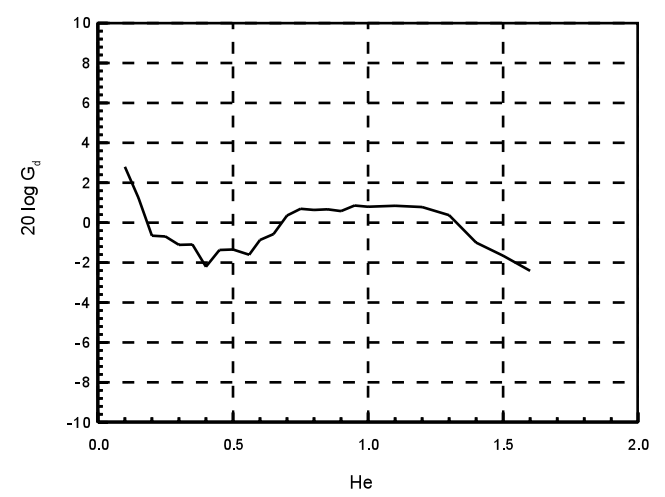

(a) Comparison of curves $S t=$ const. from Fig. 6

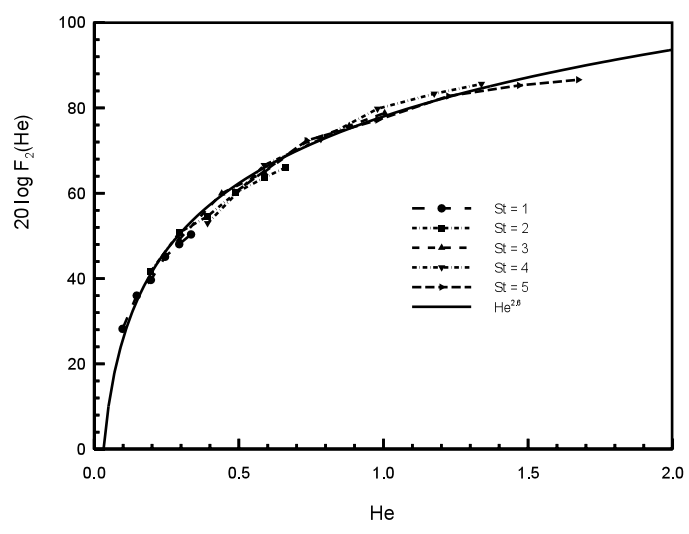

(b) System frequency characteristics

Fig. 8 Comparison of curves $S t=$ const. from Fig. 6

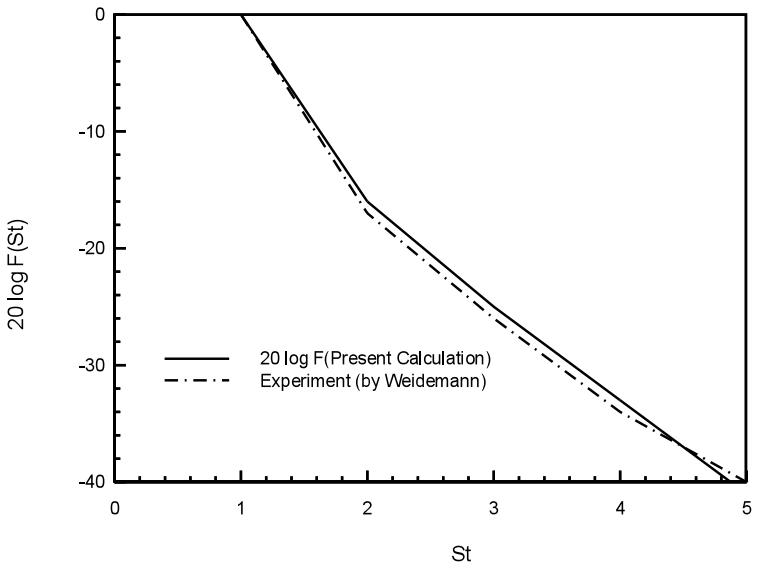

Fig. 9 Average level differences between the curves $S t=$ const. from Fig. 6

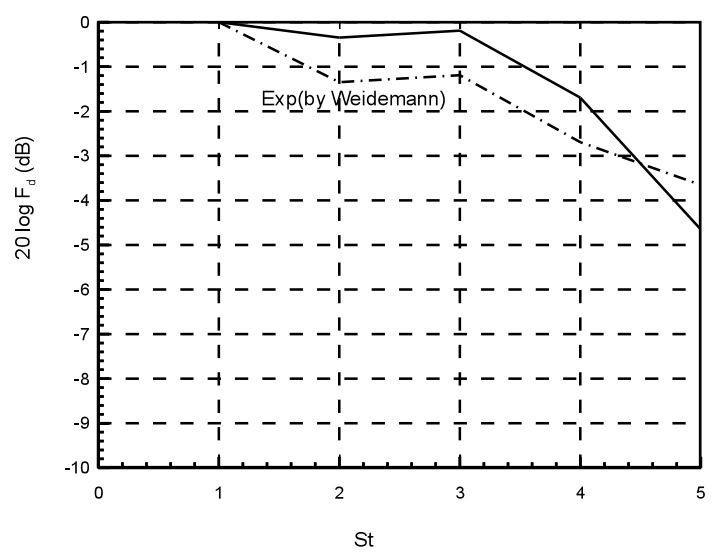

Fig. 10 Calculated and measured $F$ function (Line: Calculated, Dashdot: Experiment by Weidemann)

Now, by considering Eq. (12) we obtain the following equation:

$$
K=k_{d} M a^{\alpha} \operatorname{Re}^{\beta} F(S t) G(H e)
$$

To complete this equation we need to determine the unknown constant $k_{d}$. In order to calculate $k_{d}$ in Eq. (18), $H e=1$ is used for $S t=3$ case. Then, $R e, M a$, and $K$ are $1.12 \times 10^{6}, 0.174$ and $78.8 \mathrm{~dB}$, respectively. Next, from
Figs. 8 and 9, the $F$ and $G$ values are obtained as follows:

$$
\begin{aligned}
& F_{d}(S t=3)=0.978, \\
& G_{d}(H e=1)=1.1 .
\end{aligned}
$$

Substituting these values into Eq. (18), $k_{d}=0.954 / P_{0}$, where $P_{0}$ is the standard atmospheric pressure, is obtained. Finally, the similarity law is expressed as the following equation:

$$
\begin{aligned}
20 & \log \left[P / P_{r e f}\right] \\
& =20 \log \left(k_{d} P_{0} / P_{r e f}\right)+20 \log \left(M a^{2.6} R e^{0.2}\right) \\
& +20 \log F_{d}+20 \log G_{d} \\
& =93.5 \mathrm{~dB}+20 \log \left(M a^{2.6} R e^{0.2}\right) \\
& +20 \log F_{d}+20 \log G_{d}
\end{aligned}
$$

As an application example of Eq. (20), we want to calculate the 4th peak value of the noise generated from a centrifugal fan rotating at $3000 \mathrm{rpm}$, that is, $\mathrm{He}=1.0$ and $S t=4$. Substituting $M a=0.13$ and $R e=0.837 \times 10^{6}$ into Eq. (20) yields $70.2 \mathrm{~dB}$, which is sufficiently close to $71.5 \mathrm{~dB}$ calculated by numerical analysis. As a result, the similarity law in Eq. (20) is verified. This similarity law can be applied to both experimental and numerical cases. In reality, the noise source analysis of a centrifugal fan by experiments is difficult due to various reasons. The methodology developed in this paper can be effectively applied to determine $F_{1}(S t)$ and $F$ functions, which are related to the sound generation, although $G$ function can not be predicted because it is related to the reflection and scattering of sound, and resonance. Therefore, the proposed method can be effectively used to investigate the sound generation by centrifugal fans.

\section{Conclusions}

A new methodology to analyze noises by centrifugal fans was developed, and applied to the system for which Weidemann performed an experiment. Lowson's equation was used to calculate noises of the centrifugal fan. Flow field analysis was also performed to obtain the flow field 
data. Discrete Vortex Method was used to analyze the unsteady flow field up to 20 non-dimensional time. The calculated acoustic pressure at $3000 \mathrm{rpm}$ was satisfactorily consistent with the experimental result. The dependence of the acoustic pressures on the rpm, i.e., the impeller tip speed, was also investigated by varying rpm from 1200 to 4100 . The analysis result was consistent with the experimental one. It was also verified that the similarity law is applicable to the numerical analysis method by obtaining $F$ and $G$ functions. Although the proposed method does not consider the effects of reflection scattering, and resonance - therefore, obtain $G$ function deviated from experiments value - it can predict very reasonably $F_{1}\left(S_{t}\right)$ and $F$ functions, which are related to the sound generation. Therefore, the proposed method can perform the noise source identification satisfactorily.

\section{References}

( 1 ) Neise, W., Noise Reduction in Centrifugal Fans: A Literature Survey, Journal of Sound and Vibration, Vol.45, No.3 (1976), pp.375-403.

(2) Neise, W., Review of Noise Reduction Methods for Centrifugal Fans, J. of Engineering for Industry, Vol.104 (1982), pp.151-161.

( 3 ) Embleton, T.F., Experimental Study of Noise Reduction in Centrifugal Blowers, Journal of the Aeroacoustical Society of America, Vol.35 (1963), pp.700-705.

( 4 ) Lyons, L.A. and Platter, S., Effect of Cut-off Configuration on Pure Tones Generated by Small Centrifugal Blowers, Journal of the Aeroacoustical Society of America, Vol.35 (1963), pp.1455-1456.

( 5 ) Neise, W. and Koopmann, G.H., Reduction of Centrifugal Fan Noise by Using Resonators, Journal of Sound and Vibrartion, Vol.73, No.2 (1980), pp.297-308.

(6) Koopmann, G.H., Fox, D.J. and Neise, W., Active Source Cancellation of the Blade Tone Fundamental and Harmonics in Centrifugal Fans, Journal of Sound and Vibration, Vol.126, No.2 (1988), pp.209-220.

( 7 ) Neise, W., Application of Similarity Laws to the Blade Passage Sound of Centrifugal Fans, Journal of Sound and Vibration, Vol.43, No.1 (1975), pp.61-75.

( 8 ) Maling, G.C., Dimensional Analysis of Blower Noise, Journal of the Aeroacoustical Society of America, Vol.35 (1963), pp.1556-1564.

(9) Chanaud, R.C., Aerodynamic Sound from CentrifugalFan Rotors, Journal of the Aeroacoustical Society of America, Vol.37 (1965), pp.969-974.

(10) Weidemann, J., Analysis of the Relations between Acoustic and Aerodynamic Parameters for a Series of Dimensionally Similar Centrifugal Fan Rotors, NASA TT F-13, No.798 (1971).

(11) Neise, W. and Barsikow, B., Acoustic Similarity Laws for Fans, Trans. of the ASME, Vol.104 (1982), pp.162168.

(12) Bent, P.H., McLaughlin, D.K. and Thompson, D.E., The Influence of Discharge Configuration on the Generation of Broadband Noise in Centrifugal Turbomachinery, DGLR/AIAA 92-02-099, (1992), pp.607-614.

(13) Mongeau, L., Thomson, D.E. and McLaughlin, D.K., Sound Generation by Rotating Stall in Centrifugal Turbomachines, Journal of Sound and Vibration, Vol.163, No.1 (1993), pp.11-30.

(14) Tetu, L.G., Thompson, D.E. and McLaughlin, D.K., Aeroacoustic Similarity of Centrifugal Turbomachinery of Different Geometries, AIAA 93-4371, (1993).

(15) Jeon, W.-H. and Lee, D.-J., An Analysis of Sound Field of a Centrifugal Fan with Volute Casing, 6th AIAA Aeroacoustics Conference, AIAA/CEAS 2000-2092, (2000).

(16) Jeon, W.-H. and Lee, D.-J., An Analysis of the Flow and Aerodynamic Acoustic Sources of a Centrifugal Impeller, Journal of Sound and Vibration, Vol.222, No.3 (1999), pp.505-511.

(17) Lowson, M.V., The Sound Field for Singularities in Motion, Proc. R. Soc. London, Ser. A., Vol.286 (1965), pp.559-572. 